\title{
Variante comportamental da demência frontotemporal: relato de caso
}

Sónia Moreira, ${ }^{1-2}$ Sara Duarte, ${ }^{3-4}$ Isabel Moreira, ${ }^{3-4}$ Ernestina Santos ${ }^{4-5}$

\begin{abstract}
RESUMO
Introdução: A demência frontotemporal corresponde a um conjunto de síndromas demenciais que têm em comum alterações de personalidade, comportamento e linguagem como características clínicas precoces; e a atrofia do córtex frontal e temporal como característica anátomo-patológica. Esta entidade parece estar subdiagnosticada, pelo que se pretende sensibilizar para as dificuldades na sua identificação e na gestão terapêutica.

Descrição do caso: Homem de 74 anos sem antecedentes pessoais e familiares de relevo para a história da doença atual. Um ano após a idade normal de reforma começou a apresentar alteração progressiva dos padrões de conduta social, com ausência do controlo do impulso, associado a comportamentos alimentares desadequados e a rituais, bem como um discurso empobrecido. Algum tempo após o início dos sintomas e observação por outras especialidades médicas foi avaliado e orientado por neurologia.

Comentário: O médico de família pode ter um papel importante na suspeição desta entidade clínica, devido à prestação de cuidados longitudinais que assegura um vasto conhecimento dos utentes. Nesse sentido possibilitará uma investigação e orientações adequadas e atempadas, evitando tratamentos desnecessários e potencialmente prejudiciais. Destaca-se, ainda, o seu papel de gestão da multimorbilidade associada e de suporte aos familiares/cuidadores.
\end{abstract}

Palavras-chave: Demência frontotemporal;Variante comportamental da demência frontotemporal; Critérios de diagnóstico; Tratamento; Cuidados primários.

\section{INTRODUÇÃO}

P erante um doente em idade de reforma com sintomas neuropsicológicos de alteração de personalidade, de comportamento e/ou de linguagem, o médico de família (MF) coloca como hipóteses diagnósticas outras entidades autolimitadas e institui tratamentos em conformidade. O diagnóstico de uma síndroma demencial, nomeadamente a demência frontotemporal (DFT), não deve ser descurado, após despistadas outras etiologias e tendo em conta a sua prevalência neste grupo etário. ${ }^{1-2}$ Esta corresponde a um conjunto de entidades que têm em comum os sintomas enumerados como característica clínica precoce e a atrofia do córtex frontal e temporal como característica anátomo-patológica. ${ }^{3-4}$

1. Médica Interna de Medicina Geral e Familiar

2. USF de Fânzeres, ACeS de Gondomar

3. Médica Interna de Neurologia

4. Hospital Geral de Santo António, Centro Hospitalar do Porto

5. Médica Assistente de Neurologia
A apresentação deste caso clínico permitirá divulgar os aspetos chave para a sua suspeição e evidenciar as dificuldades associadas ao seu diagnóstico. A realização de uma história clínica adequada, não só com o doente, mas sobretudo com a família/cuidador(es) do doente é um elemento chave para estabelecer o diagnóstico. A referenciação precoce à neurologia e uma boa articulação com a medicina geral e familiar são aspetos fundamentais para assegurar uma melhor resposta, não só às necessidades do doente, mas às dificuldades que poderão surgir no seio familiar ao longo da evolução da doença. ${ }^{3-}$ 5

\section{DESCRIÇÃO DO CASO}

Homem de 74 anos, caucasiano, licenciado em engenharia, pertencente a família nuclear, na fase VIII do ciclo de vida de Duvall e na classe I de Graffar, sem antecedentes familiares de doença neurológica (Figura 1).

Reformou-se em 2007, com 65 anos, mas continuava a ter uma vida ativa e saía de casa todos os dias. Cerca de 




Figura 1. Genograma familiar com psicofigura de Mitchell.

um ano depois começou a ter algumas atitudes fora do seu habitual, por exemplo: dava sempre dinheiro a mais ao pagar no restaurante onde almoçava; os familiares começaram a notar que estava sempre a ver as horas nos relógios, mas não sabia responder quando lhe perguntavam as horas. Apesar de um dos seus passatempos favoritos ser o de assistir a jogos de futebol, os amigos referiam que o doente saía do estádio de futebol cerca de dez minutos após o início do jogo. Deixou igualmente de frequentar o ginásio, não conseguia fazer uma aula até ao fim, fartava-se e desistia rapidamente, abandonando a aula.

A mulher conta que, após a reforma, "o mundo dele parou" (sic); gradualmente foi ficando cada vez mais desmotivado, sem interesse para nada e deixou de se preocupar com a gestão da casa, nomeadamente com os pagamentos das contas da família. Aos 67 anos recorreram a um psiquiatra por suspeitarem de uma depressão. Foi medicado com antidepressivos sem apresentar melhoria clínica.

Entretanto, a mulher constatou que o doente tinha gastos excessivos e despropositados. Comprava CD de música repetidos, que nem chegava a abrir; inúmeras enciclopédias, contudo, algumas vezes repetidas; muitas camisolas iguais, que nem chegava a usar. Muitos destes artigos estavam escondidos na mala do carro, cobertos com uma manta. Além disso, descobriu que o marido passou a roubar pequenas coisas supérfluas no supermercado, principalmente perfumes e cremes, que escondia em casa. No verão de 2010, foram passar férias no local habi- 

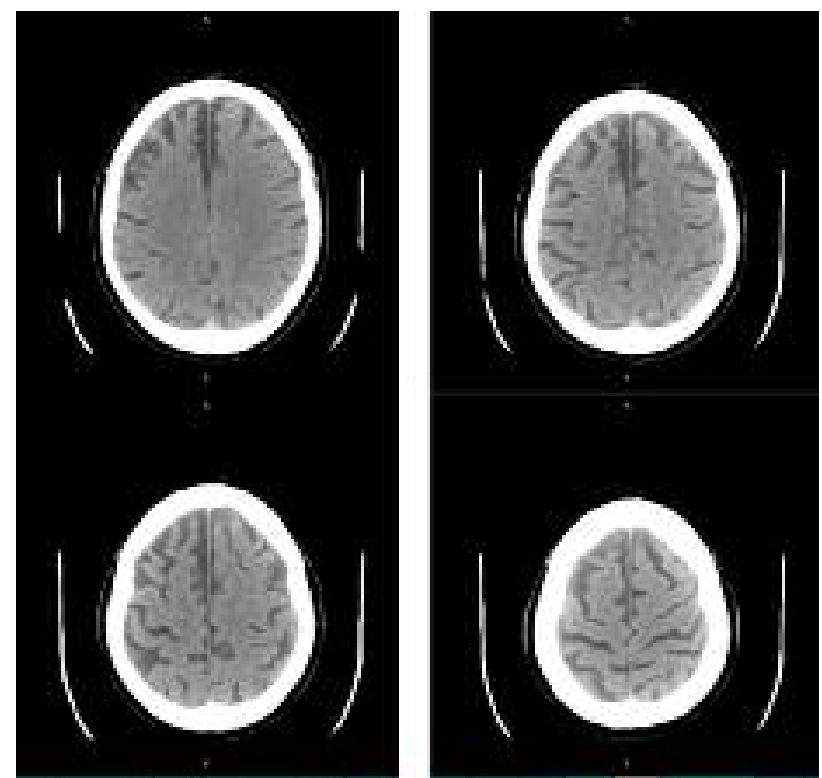

Figura 2. Cortes axiais de TC cerebral do doente (julho de 2010).

tual desde há vários anos e, durante o percurso, o doente perdeu-se duas vezes. Já no hotel desapareceu durante três dias. A polícia encontrou-o na rua, usando apenas calções de banho. Estava desidratado, pelo que foi internado e fez tomografia computorizada (TC) cerebral, que revelou atrofia dos lobos pré-frontal e temporal anterior, discretamente mais evidente à direita (lado esquerdo de cada corte axial - Figura 2).

Posteriormente começou a deixar de cuidar da sua higiene pessoal, a ter muita dificuldade em comunicar, respondia apenas quando tentavam falar com ele e não tinha iniciativa para iniciar discurso. Concomitantemente começou a ficar infantilizado, gostava muito de brincadeiras com os netos, comportando-se de igual para igual. Manteve, no entanto, sempre afeto pelos netos. Nunca demonstrou agressividade, nem teve alucinações. A mulher referiu ainda que ele apresentava total desinteresse sexual.

O seu padrão alimentar também se alterou: passando a alimentar-se de forma voraz e/ ou comendo toda a fruta que via. Perdeu o controlo dos esfíncteres. Adormecia facilmente, mas acordava pelas 5-6 horas da manhã e iniciava movimentos repetitivos e estereotipados, como bater repetidamente nas grades da cama.

No início de julho de 2012, o MF solicita observação por neurologia por suspeição de síndroma demencial grave, explicitando no pedido a situação clínica e a difi- culdade dos familiares em levarem o doente para a realização de estudo complementar alargado. Por esta altura, foi também pedido apoio do serviço social.

Na primeira observação em neurologia, em finais de julho, o doente apresentou um comportamento desadequado: esteve sempre a comer e muito inquieto, mexendo em tudo o que estava pousado na secretária. No exame neurológico apresentou afasia, de predomínio motor, que comprometeu a realização da avaliação neuropsicológica, e reflexo palmomentoniano bilateral. Estava medicado com gabapentina $100 \mathrm{mg}$ (duas vezes por dia); sertralina 50mg (uma vez por dia), donepezilo $5 \mathrm{mg}$ (uma vez por dia) e lorazepam $1 \mathrm{mg}$ (uma vez por dia). Estabeleceu-se, como provável diagnóstico, demência frontotemporal variante comportamental (DFTvc). Recomendou-se suspender a gabapentina e o donepezilo, manter a sertralina e o lorazepam, e iniciar trazodona 100mg (uma vez por dia, ao deitar) e foi agendada consulta de reavaliação, explicando-se as implicações do diagnóstico e a possível evolução da doença.

Em janeiro de 2013 há registo de internamento no serviço de endocrinologia por descompensação da diabetes mellitus tipo 2 associada a coma hiperosmolar hiperglicémico, insuficiência renal aguda e rabdomióliseuma associação provável ao aumento da ingestão de doces e inatividade física em doente com vários fatores de risco cardiovasculares.

Na consulta de neurologia subsequente, em março de 2013, a mulher referia que estava mais tranquilo, com sono regularizado e dependente para todas as atividades de vida diária. Necessitava de ajuda na alimentação, para se vestir e no banho. Ao exame neurológico apresentou-se desatento, sem iniciativa verbal e motora, sempre a esboçar uma gargalhada, rindo-se, por vezes, a despropósito. Nomeou alguns objetos com erros e cumpriu apenas ordens simples. Registou-se ainda reflexo de grasping bilateral, sucking e estereotipias das mãos.

O doente manteve acompanhamento regular com o MF, destacando-se as intervenções ao nível da vigilância: dos fatores de risco cardiovascular e, em particular, da diabetes mellitus tipo 2; da adesão à terapêutica e renovação do receituário; e na prescrição de tratamentos de medicina física e de reabilitação para promoção da autonomia funcional, em abril de 2013.

Em dezembro de 2013, na consulta subsequente de neurologia, a mulher relata que apresenta cada vez maior di- 
ficuldade em se expressar, "agora só abana cabeça sim-não" (sic) em sinal de resposta. O doente passou a consulta toda a mascar pastilha elástica e, segundo a mulher, se lhe retirasse a pastilha elástica, passaria o tempo a bater com as mãos na mesa. Há ainda referência a desorientação dentro da própria habitação: quando a mulher lhe dizia para ele ir ao quarto de banho, ele "ia para a sala" (sic). Não revelava comportamentos agressivos, nem alucinações.

Em março de 2014, há referência a episódios de agressividade, de curta duração, após a higiene e mantinha hiperfagia, pelo que se decidiu introduzir topiramato e otimizar a dose de trazodona para 300mg (uma vez dia, ao deitar) e manter restante medicação. ATC cerebral (Figura 3), realizada em data próxima da consulta de seguimento, demonstrou achados concordantes com o diagnóstico clínico e agravamento da atrofia frontotemporal.

Cerca de um mês após a consulta é solicitada visita domiciliária ao MF, porque o doente apresenta deterioração importante da sua funcionalidade e há referência a dificuldade de deglutição. Nesta fase de evolução da doença são notórios os sinais indicativos de exaustão da cuidadora (mulher), evidenciando sintomatologia depressiva reativa e dificuldade na aceitação da doença do marido.

Em dezembro de 2014 obtiveram-se os resultados dos biomarcadores do líquido cefalorraquidiano (presença de proteína tau elevada e beta amiloide normal), solicitados pela neurologia. Estes revelaram-se concordantes com o diagnóstico de DFTvc. Segundo a mulher, dorme e alimenta-se bem, com episódios de engasgamento; usa sempre fraldas; passa o dia calmo, quase não fala, não lê, nem escreve. Mantém estereotipias frequentes: está sempre a bater palmas, nas pernas ou na mesa.

A última consulta de neurologia decorreu em março de 2016, denotando-se um agravamento da doença, estando o doente mais debilitado, com ausência completa de peças dentárias, mas com melhoria das dificuldades na deglutição. Já não comunica, nem reconhece os familiares e amigos. A mulher reporta que tem medo da água, assustando-se com o toque, e mantém-se como cuidadora principal, com escasso apoio dos restantes familiares.

No intervalo entre estas consultas decorreram visitas domiciliárias regulares do MF para apoio na gestão das intercorrências (e.g., obstipação; edema dos membros inferiores) e dos problemas crónicos, bem como no suporte aos cuidadores.


Figura 3. Cortes axiais de TC cerebral do doente (julho de 2010).

\section{COMENTÁRIO}

A evolução dos factos narrados retrata a dificuldade na orientação diagnóstica e terapêutica da DFT. Desde a evidência dos primeiros sintomas até ao diagnóstico decorreram cerca de cinco anos, que envolveram várias observações por diferentes médicos de diferentes especialidades, provas terapêuticas com a possibilidade de efeitos secundários associados, pedidos de exames complementares e o sofrimento inerente à incerteza no diagnóstico.

Em termos epidemiológicos, constitui a segunda causa mais comum de demência antes dos 65 anos (a seguir à doença de Alzheimer), com uma prevalência estimada de 15-22 casos por 100.000 pessoas entre os 45 e os 65 anos. A DFTvc é o subtipo mais prevalente. A nível nacional desconhece-se a sua prevalência, mas os estudos referem que poderá estar subdiagnosticada. ${ }^{3-4,6}$ Este facto tem implicações importantes em termos da qualidade dos cuidados que podem estar a ser prestados a estes doentes.

Na observação pela neurologia, em 2012, foi possível apurar caraterísticas clínicas preponderantes, bem como informações sobre as orientações prévias fornecidas ao doente e à mulher, principal cuidadora. A reflexão sobre estes dados permitiu considerar a hipótese de DFTvc como muito provável. 
A apresentação clínica caracterizou-se por instalação progressiva de alteração dos padrões de conduta social (roubos no supermercado, mexer em coisas alheias e comer durante a consulta), ausência de controlo do impulso e perda da autocrítica (as compras excessivas e desnecessárias; roubos no supermercado), bem como pela apatia. Registou-se também impersistência nas tarefas (incapaz de se manter na aula no ginásio ou no jogo de futebol até ao fim); alteração do comportamento alimentar, com hiperfagia (preferência por doces); e comportamentos ritualizados (sempre a olhar para o relógio) e aditivos (sempre a ler enciclopédias). Adicionalmente a estas características apresentava estereotipias (bater com as mãos nas grades da cama), comportamento de utilização (pegar nos papéis e canetas em cima da secretária durante a consulta) e presença de reflexos primitivos. De destacar, ainda, as alterações da linguagem, com uma redução progressiva da produção do discurso. Numa primeira fase há referência a um discurso estereotipado, com economia da linguagem, e presentemente encontra-se numa fase de quase mutismo e imobilidade.

Em termos gerais, as alterações do comportamento e da linguagem são os aspetos dominantes deste tipo de demência e as características base comuns aos vários subgrupos da DFT. ${ }^{3-4,7}$ Os problemas de memória aparecem mais tardiamente, tornando mais difícil o diagnóstico da síndroma demencial. Do ponto de vista cognitivo verifica-se uma deterioração dominante das capacidades executivas (planeamento, sistematização) e da atenção, que muitas vezes só é detetada após uma avaliação neuropsicológica detalhada. Nos exames neuropsicológicos, tanto funcionais como estruturais, os doentes afetados podem apresentar resultados normais ou levemente patológicos. Estes exames assumem, assim, uma importância extraordinária, pois identificam e separam as funções cognitivas deterioradas das intactas. Contudo, à semelhança do que ocorreu na avaliação do doente, as funções cognitivas são, por vezes, difíceis de avaliar devido às alterações emocionais do doente, à eventual disfunção da linguagem e/ou a um estadio avançado da doença. . $^{3-46-7}$

Relativamente ao estudo imagiológico, é obrigatória a sua realização na avaliação inicial de um doente com clínica sugestiva de DFTvc de forma a serem excluídas alterações estruturais, nomeadamente patologia tumoral ou doença vascular cerebral. Adicionalmente, permite compreender o estadio da doença, uma vez que ao lon- go da evolução da doença ocorre uma progressiva atrofia das porções anteriores dos lobos frontais e temporais (como observado nas imagens de TC cerebral das Figuras 2 e 3). Este achado, por não ser objetivável nos estadios iniciais, pode justificar a referenciação precoce para os cuidados de saúde secundários para prosseguir estudo com análise volumétrica por ressonância magnética cerebral e/ou estudo de imagem funcional. ${ }^{4,6-7}$ Neste caso foi possível o acesso às imagens do TC cerebral realizado na primeira admissão hospitalar, com caráter urgente, e que teve como objetivo prioritário a exclusão de patologias agudas. Este dado pode explicar, em parte, a não valorização do achado imagiológico de atrofia cerebral. Em 2014, o pedido de TC cerebral de reavaliação permitiu a realização de uma análise de confrontação.

De notar que, apesar de a semiologia ser muito sugestiva de DFTvc, o diagnóstico definitivo é anátomo-patológico. As alterações histológicas encontram-se nas porções anteriores dos lobos frontais e temporais e, em alguns núcleos, da base do cérebro. Descrevem-se classicamente perda de neurónios, degenerescência sináptica, gliose e diferentes inclusões neuronais (como os corpos de Pick). Existe, assim, uma correlação entre as alterações genéticas, o fenótipo clínico e a patologia na DFT, que tem sido utilizada na classificação ainda em aperfeiçoamento desta doença. ${ }^{7-9}$

Apesar de neste caso não se ter apurado existência de história familiar (Figura 1), em cerca de $25-50 \%$ dos casos pode existir história familiar de DFT, sendo usualmente de transmissão autossómica dominante. Nesse sentido, perante a apresentação clínica sugestiva de DFT, o estudo de antecedentes familiares não deve ser descurado. ${ }^{6,8-9}$

$\mathrm{Na}$ abordagem inicial foram excluídas as possibilidades de estar perante sintomas psicológicos ou comportamentais (agitação, agressividade, depressão e/ou ansiedade, desinibição e euforia ou apatia, insónia) de uma etiologia orgânica (como infeções respiratória, urinária, entre outras; descompensação de patologia de base; alterações hidroeletrolíticas); ou resultantes de efeitos laterais (efeitos colinérgicos; outros tóxicos) ou de abstinência de fármacos.

Por outro lado, nas observações médicas prévias do doente, foram despistados aspetos clínicos que colocavam em causa o diagnóstico, nomeadamente: início súbito da sintomatologia (hipótese de depressão); a história de traumatismo cranioencefálico a preceder os sintomas; a perda de memória marcada no estadio inicial; 


\section{QUADRO I. Manifestaçōes clínicas ${ }^{3 \cdot 4,7}$}

Alterações do comportamento, | Desinibição com jocosidade inapropriada, perda de autocrítica e/ou da conduta social, ou do humor e da personalidade apatia, desinteresse; comportamentos repetitivos; hiperfagia; labilidade emocional - crise de depressão tardia

Alterações da linguagem

Alterações das funções executivas e da atenção

Alterações da memória
Discurso empobrecido, estereotipado, associado a parafasias

Planeamento, memória de trabalho, abstração, flexibilidade mental, solução de problemas

Tardias o predomínio da desorientação espacial ou a presença de sinais piramidais no exame neurológico. Considera-se importante excluir outras patologias que podem originar um quadro demencial com características clínicas que simulam DFT ou excluir a possibilidade de estar perante uma outra demência (como a demência vascular).

O estudo laboratorial e/ou imagiológico permitiu excluir patologias que podem atingir o sistema nervoso central, nomeadamente: patologia infecciosa (neurossífilis; doença de Creutzfeldt-Jakob; encefalopatia por vírus da imunodeficiência humana); tumores do lobo frontal, esclerose múltipla, entre outros..$^{8,10}$

Não se dispõe, até ao momento, de terapêutica específica aprovada para a DFT, apesar dos novos conhecimentos sobre a genética da DFT que podem ter importantes implicações no tratamento. Atualmente, a instituição de terapêutica destina-se ao controlo sintomático. O doente foi medicado inicialmente com donepezilo, sem benefício terapêutico. Os inibidores das colinesterases (donepezilo, memantina) têm sido usados de forma empírica, mas sem eficácia comprovada. ${ }^{3-4,10}$ Além disso, o donepezilo pode ter um efeito paradoxal de agravamento da agitação e da impulsividade, recomendando-se a sua suspensão.

Em relação à terapêutica destinada a estabilizar as alterações comportamentais, em particular a agitação, vários fármacos têm sido tentados, nomeadamente os neurolépticos atípicos e os antiepilépticos, mas sem resultados sustentados, podendo os primeiros agravar os sintomas parkinsónicos. As benzodiazepinas não estão indicadas na maioria das situações, podendo induzir agitação paradoxal e efeitos cognitivos negativos.

Relativamente aos fármacos inibidores da recaptação da serotonina (como a sertralina) têm-se registado resultados nos casos que apresentam sintomas depressivos, comportamentos estereotipados e ritualísticos ou desinibição. Nesse sentido considera-se adequada a sua manutenção na primeira avaliação clínica realizada.

Aliada à terapêutica farmacológica existem algumas medidas que poderão melhorar a qualidade de vida destes doentes. No caso em concreto, o MF recomendou a realização de tratamentos de reabilitação. Poderia ainda ter sido integrado em programas de terapia da fala, devido às perturbações da linguagem. No atual estadio da DFTvc deve-se procurar evitar alterações bruscas do ambiente (como mudança de cuidador ao fim-de-semana; volume de televisão muito alto; excesso de estimulação), adotando uma atitude não confrontativa nem recriminativa., ${ }^{3,5,10}$

Contudo, apesar de ser possível alguma estabilidade clínica com recurso a técnicas ambientais e comportamentais, associadas ao tratamento farmacológico, trata-se de uma doença progressiva, grave e muito incapacitante.

Um dado interessante é que estes doentes recorrem mais precocemente à consulta de medicina geral e familiar, comparativamente com doentes com provável diagnóstico de doença de Alzheimer, o que talvez tenha relação com a idade de início mais precoce e pelo facto destas alterações serem pior toleradas.

Estas características são reportadas frequentemente por/pelos familiar(es) do doente e devem alertar o MF. Durante a consulta poderá também detetar alterações da linguagem associadas (como discurso empobrecido) e, eventualmente, não tão valorizadas pelo doente e/ou familiar(es). Estes dois aspetos, aliados a uma preservação relativa da memória e da orientação topográfica (são doentes que não se perdem até um estadio avançado e que reconhecem sempre as pessoas, incluindo o MF), são os aspetos mais importantes para a suspeição do diagnóstico.4-5

Os critérios de diagnóstico clínico mencionados (Quadro I) apresentam uma especificidade e uma sensibilidade razoável e os exames subsidiários, embora possam 
ajudar a excluir situações que impossibilitam o diagnóstico, não são críticos para o seu estabelecimento. ${ }^{1-2,7}$ Por essa razão, perante um doente com apresentação sugestiva de DFT, é importante o MF explorar adequadamente as suas características, colocar como hipótese de diagnóstico e referenciar atempadamente a neurologia, evitando investigação e tratamentos desnecessários e potencialmente prejudiciais. Considera-se ainda importante destacar o papel do MF na gestão da multimorbilidade associada, na prestação de cuidados domiciliários em fases avançadas da doença e de suporte ao(s) familiar(es) e/ou cuidador(es) do doente.

\section{REFERÊNCIAS BIBLIOGRÁFICAS}

1. Neary D, Snowden JS, Gustafson L, Passaut U, Stuss D, Black S, et al. Frontotemporal lobar degeneration: a consensus on clinical diagnostic criteria. Neurology. 1998;51(6):1546-54.

2. McKhann GM,Albert MS, Grossman M, Miller B, Dickson D, Trojanowski JQ. Clinical and pathological diagnosis of frontotemporal dementia: report of the work group on frontotemporal dementia and Pick's disease. Arch Neurol. 2001;58(11):1803-9.

3. Karageorgiou E, Miller BL. Frontotemporal lobar degeneration: a clinical approach. Semin Neurol. 2014;34(2):189-201.

4. Pelicano-Paulos J, Massano J. Clinical, genetic and neuropathological features of frontotemporal dementia: an update and guide. Acta Med Port. 2013;26(4):392-401.

5. Alves RP, Caetano Al. O papel do médico de família no diagnóstico e seguimento dos doentes com declínio cognitivo e demência [The role of the family physician in the diagnosis and monitoring of patients with cognitive decline and dementia]. Rev Port Clin Geral. 2010;26(1):6974. Portuguese

6. Guimarães J, Fonseca R, Garrett C. Demência frontotemporal: que entidade? [Frontotemporal dementia]. Acta Med Port. 2006;19(4):31924. Portuguese

7. Rascovsky K, Hodges JR, Knopman D, Mendez MF, Kramer JH, Neuhaus J, et al. Sensitivity of revised diagnostic criteria for the behavioural variant of frontotemporal dementia. Brain. 2011;134(Pt 9):2456-77.

8. Taipa R, Pinho J, Melo-Pires M. Clinico-pathological correlations of the most common neurodegenerative dementias. Front Neurol. 2012;3:68.

9. Seelaar H, Rohrer JD, Pijnenburg YA, Fox NC, Van Swieten JC. Clinical, genetic and pathological heterogeneity of frontotemporal dementia: a review. J Neurol Neurosurg Psychiatry. 2011;82(5):476-86.

10. Couto FS, Mendonça A. Sintomas psiquiátricos e comportamentais da demência [Behavioral and psychiatric symptoms of dementia]. Rev Port Clin Geral. 2010;26(1):55-60. Portuguese

\section{CONFLITOS DE INTERESSE}

As autoras declaram não possuir qualquer tipo de conflitos de interesse.

\section{ENDEREÇO PARA CORRESPONDÊNCIA}

Sónia Moreira

USF de Fânzeres

Praceta da Barrosa, s/n

4510-503 Fânzeres

E-mail: soniamarquesmoreira@gmail.com

Recebido em 31-05-2016

Aceite para publicação em 24-02-2017

\section{ABSTRACT}

\section{BEHAVIORAL CHANGES IN FRONTOTEMPORAL DEMENTIA: A CASE REPORT}

Introduction: Frontotemporal dementia is characterized by changes in personality, behavior, and language. Atrophy of the frontal and temporal cortex is the main pathological finding. As this entity is under-diagnosed, it is important to raise awareness of the difficulties in diagnosis and management.

Case Description: A 74 year-old man had progressive changes in appropriate social behavior from age 66. This included a lack of impulse control, inadequate food behaviors and rituals, and reduced production of speech. Some time after the onset of symptoms and consultation with different medical specialists, he was referred to a neurologist who made the diagnosis of frontotemporal dementia. Commentary: The family physician can play an important role by suspecting this condition, through longitudinal care and personal knowledge of the patient. Proper investigation and appropriate and timely guidance can help avoid unnecessary and potentially harmful treatments. The family physician can also manage associated comorbidity and support the family and other caregivers.

Keywords: Frontotemporal Dementia; Behavioral variant of Frontotemporal Dementia; Diagnosis; Therapeutics; Primary Care. 